\title{
Outcomes of Cognitively Impaired Not Demented at 2 Years in the Canadian Cohort Study of Cognitive Impairment and Related Dementias
}

\author{
Ging-Yuek R. Hsiung ${ }^{\mathrm{a}} \quad$ Alan Donald $^{\mathrm{b}}$ Jacob Grand ${ }^{\mathrm{a}} \quad$ Sandra E. Black $^{\mathrm{c}}$ \\ Remi W. Bouchard $^{d}$ Serge G. Gauthiere Inge Loy-English ${ }^{f}$ David B. Hogan ${ }^{9}$ \\ Andrew Kertesz $^{\text {h }}$ Kenneth Rockwood ${ }^{i}$ Howard H. Feldman ${ }^{a}$ \\ a Division of Neurology, Department of Medicine University of British Columbia, ${ }^{\mathrm{b}}$ Department of Statistics,

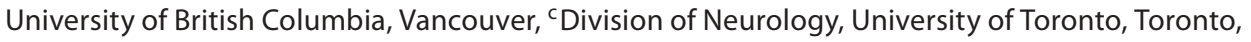 \\ ${ }^{\mathrm{d}}$ Division of Neurology, Laval University, Quebec City, ${ }^{e}$ Departments of Neurology and Neurosurgery, \\ Psychiatry, Medicine, McGill University, Montreal, ${ }^{f}$ Division of Neurology, University of Ottawa, Ottawa, \\ gDivision of Geriatric Medicine, University of Calgary, Calgary, ${ }^{\mathrm{h}}$ Division of Clinical Neurological Sciences, \\ University of Western Ontario, London, 'Divisions of Geriatric Medicine and Neurology, Department of Medicine, \\ Dalhousie University, Halifax, Canada
}

\section{Key Words}

Dementia $\cdot$ Cognitively impaired not demented .

Mild cognitive impairment $\cdot$ Alzheimer's disease $\cdot$

Longitudinal cohort $\cdot$ ApoE

\begin{abstract}
Background: People who are cognitively impaired not demented (CIND) can be at an increased risk for developing dementia, but little is known about the natural history of CIND in clinical settings. Method: We examined the 2-year outcome of CIND subjects in the Canadian Cohort Study of Cognitive Impairment and Related Dementias. CIND was diagnosed when at least one positive item was endorsed on the DSM-III-R dementia criteria, but not all criteria were met. CIND was further subclassified as: pre-Alzheimer's disease (pre-AD), vascular cognitive impairment (VCI-ND), non-AD degenerative, psychiatric, other neurologic, other medical conditions, mixed disorders and no etiology identified (not otherwise specified [NOS]). Result: Of 146 CIND patients with 2-year follow-up data available, 49 (34\%) progressed to dementia, while $20(14 \%)$ recovered to not cognitively im-
\end{abstract}

\section{KARGER}

Fax +41613061234 E-Mail karger@karger.ch www.karger.com (c) 2006 S. Karger AG, Basel

$1420-8008 / 06 / 0226-0413 \$ 23.50 / 0$

Accessible online at:

www.karger.com/dem paired $(\mathrm{NCl})$. Progressors were significantly older than stable CIND and reverters $(p<0.0001$; mean age $=71.1,64.3$, and 59.1 , respectively), and there were significantly $(p=0.001)$ more ApoE $\varepsilon 4$ carriers among progressors (67\%) than stable CIND (29\%) and reverters (12\%). Pre-AD CIND and VCI-ND had the highest rate of conversion to dementia (41.0 and $40.0 \%$, respectively), while psychiatric CIND and CIND NOS had highest rate of recovery to $\mathrm{NCl}(20.0$ and $30.0 \%$, respectively). All conversions in pre-AD CIND were to 'probable AD'. Conclusion: CIND consists of a heterogeneous group of disorders that can be classified syndromically. Many subclassess - not just those with pre-AD CIND - are at high risk of progression to dementia, usually to Alzheimer's disease.

Copyright $\odot 2006$ S. Karger AG, Basel

\section{Introduction}

There is considerable interest in examining the characteristics of the earliest stages in dementia. Changes in cognitive function occur in a continuum from normal aging to dementia, but progression is neither inevitable

Dr. Howard H. Feldman

Division of Neurology, University of British Columbia

S192 - 2211 Wesbrook Mall

Vancouver, BC (Canada) V6T 2B5

Tel. +1 604822 7697, Fax +1 604822 7703, E-Mail hfeldman@interchange.ubc.ca 
nor uniform. Various constructs have been used to define the intermediate stage between normal aging and dementia. Mild cognitive impairment (MCI) depicts subjects with demonstrable impairment in cognitive function who otherwise perform normally on instrumental activities of daily living. MCI criteria initially emphasized memory difficulties [1], and was conceptualized as a precursor to Alzheimer's disease (AD). This was soon revised to reflect that cognitive domains other than memory can be impaired, so that the definition of MCI now includes impairment in non-memory domains and in multiple domains [2]. Another term, age-associated memory impairment (AAMI), has been used to depict elderly subjects with memory complaints substantiated by impairment in tests of recent memory in comparison to norms [3]. However, this broad concept includes individuals who score 1 standard deviation below norms of 'young adults', but may still be considered within the norm for 'older adults' who are otherwise functioning normally in their daily lives $[4,5]$. Another construct, age-associated cognitive decline, has been developed to identify individuals who experience cognitive decline but fall short of dementia, and includes people with impairment in memory and learning, attention and concentration, problem solving, abstraction, language, and visuospatial functioning, in relation to healthy individuals of the same age and level of education [6]. In population epidemiologic studies, the term 'cognitive impairment with no dementia' (CIND) [7] has been used to describe subjects who have some positive items on the DSM-III-R [8] checklist (Appendix 1) for diagnosis of dementia, but do not fulfill all the criteria for dementia. In the Canadian Study of Health and Aging, it was estimated that $16.8 \%$ of the population over 65 years old suffer from CIND, twice the prevalence of dementia [9]. The diagnosis of CIND has the advantage of being inclusive and comprehensive, spanning the spectrum between normal cognition and dementia, and includes all the categories from which other groupings (amnestic MCI, single-domain and multi-domain MCI, more severe forms of AAMI when memory impairment was clinically significant for age, age-associated cognitive decline) are derived. It is potentially applicable to both epidemiologic and clinical research $[10,11]$. However, it is heterogeneous and includes a variety of disorders which may or may not progress to dementia. Little is known about the natural history of CIND in the clinical setting.

The Canadian Cohort Study of Cognitive Impairment and Related Dementias (ACCORD) is a prospective longitudinal cohort of subjects referred to eight dementia clinics across Canada. The current study describes the 2year outcome of subjects who were initially diagnosed as CIND at their entry to the study. Factors which may influence the progression of CIND, including demographics and ApoE carrier status, are also examined.

\section{Methods}

As detailed elsewhere [12], the ACCORD cohort consists of 1,136 patients newly referred to eight university-based dementia research clinics across Canada with annual follow-up. The patients may be referred from family physicians or other specialists. Consent was obtained at the initial visit. Demented subjects assented and co-consent was obtained from their next of kin, legal guardian, or substitute decision maker as per provincial guideline for consent to research in incompetent subjects. A separate consent was obtained for anonymous ApoE genotyping.

Site investigators diagnosed not cognitively impaired (NCI), CIND, or dementia according to criteria adapted from the DSMIII-R criteria (Appendix 1) [8]. Clinicians were asked to apply clinical judgement in determining the presence or absence of each checklist item of the DSM-III-R taking into account all available clinical, laboratory, neuroimaging and neuropsychological test data. Patients who fulfilled the full criteria of the DSM-III-R were diagnosed as demented $(n=670)$. Subjects were diagnosed NCI, if they did not fulfill any of the DSM-III-R criteria for cognitive impairment $(n=124)$. CIND was diagnosed $(n=342)$ in people with at least one positive item endorsed on the DSM III-R cognitive function checklist, but who did not otherwise meet all the DSM-III-R criteria for dementia. The CIND diagnosis was further subclassified by etiology with all available investigation data. The subclassification categories included pre-Alzheimer disease (pre-AD), vascular cognitive impairment (VCI-ND), non-AD degenerative disorders, psychiatric, other neurological, other medical conditions, mixed disorders, and no clear etiology identified (not otherwise specified [NOS]). CIND subjects who were classified as pre-AD, (referred to as amnestic CIND in the baseline/ methods paper [12]) generally had a predominant memory complaint, with no other cognitive domains significantly affected. This may include MCI (MCI-amnestic type and MCI multi-domain type with predominant amnestic features) as defined by $\mathrm{Pe}$ tersen et al. [1, 13], more severe forms of AAMI when memory impairment was clinically significant for age, and CIND with strong family history of AD (two or more affect family members with at least one first degree relative diagnosed with clinical probable or pathologically confirmed AD). Subjects who were classified as VCI-ND had a history of cerebrovascular disease, associated focal neurological signs, or a history of multiple vascular risk factors with neuroimaging evidence of large or small vessels ischemic changes in the brain. VCI-ND data were collected following a protocol for the investigation of vascular cognitive impairment, as detailed previously [14]. The non-AD degenerative CIND group included suspected syndromes of early frontotemporal dementia, Parkinson disease with dementia, Lewy body dementia, corticobasal degeneration, multiple systems atrophy, and progressive supranuclear palsy, but not yet fulfilling their respective diagnostic criteria. Patients with 'psychiatric CIND' had a previ- 
Table 1. Baseline characteristics of the CIND cohort

\begin{tabular}{lccc}
\hline Groups & $\begin{array}{l}\text { Attended 2-year } \\
\text { follow-up }\end{array}$ & $\begin{array}{l}\text { Lost to } \\
\text { follow-up }\end{array}$ & Died \\
\hline Subjects & 146 & 190 & 6 \\
Female (\% within group) & $73 \pm 50.0$ & $95 \pm 50.0$ & $3 \pm 50.0$ \\
Age & $65.8 \pm 10.4$ & $66.5 \pm 11.0$ & $81.3 \pm 6.0^{*}$ \\
Years of education & $12.4 \pm 3.8$ & $12.5 \pm 4.4$ & $10.8 \pm 4.3$ \\
MMSE & $27.5 \pm 2.1$ & $26.3 \pm 3.5^{*}$ & $25.5 \pm 3.9^{*}$ \\
CIRS & $4.8 \pm 3.5$ & $4.7 \pm 3.3$ & $7.2 \pm 4.4$ \\
FRS & $13.8 \pm 3.0$ & $14.0 \pm 4.1$ & $13.8 \pm 2.9$ \\
DAD & $93.7 \pm 10.0$ & $89.0 \pm 12.0^{*}$ & $92.0 \pm 5.7$ \\
NPI & $8.6 \pm 15.6$ & $11.4 \pm 15.2$ & $7.3 \pm 7.6$ \\
\hline
\end{tabular}

* Denotes significant differences by ANOVA and post-hoc Dunnett test with $\mathrm{p}<0.05$ compared to those who attended follow-up. ous or current diagnosis of a major psychiatric illness (i.e. depression, schizophrenia) that was felt to be responsible for their cognitive impairment. The 'other medical CIND' subcategory consisted of medical illnesses that were judged to be significantly affecting cognition such as B12 deficiency, hypothyroidism, and chronic alcohol use including Wernicke encephalopathy and Korsakoff syndrome. The category of 'other neurological CIND' included post-encephalitis, cerebral palsy, metabolic leukoencephalopathy, traumatic brain injury, multiple sclerosis, and normal pressure hydrocephalus. The 'mixed CIND' designation consisted of subjects with mixed or multiple causes of cognitive impairment. The remaining CIND patients who had no clear cause identified after extensive clinical evaluation were grouped as 'NOS'.

For those who converted to dementia, the primary cause of dementia (i.e. the cause that was clinically designated as the most significant) was further subclassified based on clinical criteria. AD was diagnosed by NINCDS-ADRDA criteria [15]. Vascular dementia $(\mathrm{VaD})$ was diagnosed based on the presence of history of stroke, neurological signs consistent with previous cerebrovascular accidents, risk factors, or neuroimaging data consistent with stroke or chronic small vessels ischemic changes. Frontotemporal dementia was diagnosed based on criteria published by the Lund and Manchester group [16]. Dementia with Lewy body (DLB) was diagnosed by criteria by McKeith et al. [17].

All patients had detailed clinical evaluations at inception and subsequent visits, including: (1) the Mini-Mental Status Examination (MMSE) [18] - used as a general measure of cognitive impairment, (2) the Functional Rating Scale (FRS) $[19,20]$ - used as a measure of global severity of dementia, and (3) the Cumulative Illness Rating Scale (CIRS) [21, 22] - used to assess co-morbid medical conditions. Other assessments, where there were available informants, included the Disability Assessment for Dementia (DAD) [23], and the Neuropsychiatric Inventory (NPI) [24]. On both the MMSE (a 30-point scale) and the DAD (a 100 point scale), a higher total score represents better performance, while for the FRS (40 points total), CIRS (maximum 96 points), and NPI (maximum 144 points), the higher the score the worse the rating. Each CIND patient was eligible to have neuropsychological testing, the result of which was not available at the time of the CIND diagnosis by the investigator, but was available at the time of etio- logical subclassification. The ACCORD data collection was comparable to the clinical assessments for the Canadian Study of Health and Aging [9], and for the Consortium to Investigate Vascular Impairment of Cognition [14].

Our study population consists of people who completed 2 years of follow-up, as well as those who were lost to follow-up. For those who completed the second year assessment, descriptive statistics were used to depict the progression and change in diagnostic category of the cohort. Chi-square test was used to compare proportions between categorical factors. Comparisons of numerical scales were made by analysis of co-variance adjusted for age, sex, and education, with the use of Student-Newman-Keuls test for post-hoc analysis. For comparison of NPI scores which increases geometrically, the natural log of the scores was used for comparison, while the actual mean of the raw scores are presented in the results tables. For the CIND subjects who were lost to follow-up or died, baseline characteristics were compared to those who completed the second assessment using ANOVA with posthoc Dunnett test.

\section{Results}

At 2-year follow-up of the 342 CIND patients, 146 had completed their second annual assessment. Six had died and 190 were lost to follow-up (table 1). In comparing the baseline characterisitics of these three groups, those who died were significantly older and had lower total MMSE and DAD scores. When the lost to follow-up group was compared to the group who completed their second assessment, the former had significantly lower total MMSE scores. Otherwise there were no significant differences between the three groups in baseline characteristics and distribution of etiologic subtypes. There were significant differences on loss to follow-up among sites ( $\mathrm{p}<0.001)$, with two sites accounting for over 50\% (96/190) of the 
Table 2. Age and baseline clinical rating scales of CIND etiologic subtypes

\begin{tabular}{|c|c|c|c|c|c|c|}
\hline CIND subtypes $(\mathrm{n}=342)$ & Age & MMSE & CIRS & $\mathrm{DAD}$ & FRS & NPI \\
\hline Pre-AD ( $\mathrm{n}=88)$ & $69.7 \pm 8.4^{\mathrm{a}, \mathrm{b}}$ & $26.6 \pm 3.4$ & $4.0 \pm 3.1^{\mathrm{b}}$ & $93.7 \pm 10.8^{\mathrm{a}, \mathrm{b}}$ & $13.0 \pm 3.3^{\mathrm{b}}$ & $3.9 \pm 5.9^{c}$ \\
\hline Vascular $($ VCI-ND) $(\mathrm{n}=66)$ & $72.1 \pm 9.8^{\mathrm{a}}$ & $26.4 \pm 3.3$ & $6.5 \pm 4.1^{\mathrm{a}}$ & $86.3 \pm 17.1^{\mathrm{b}}$ & $13.8 \pm 4.1^{\mathrm{b}}$ & $12.6 \pm 16.4^{\mathrm{b}, \mathrm{c}}$ \\
\hline Psychiatric $(\mathrm{n}=59)$ & $61.7 \pm 11.5^{\mathrm{b}}$ & $26.9 \pm 2.6$ & $5.0 \pm 2.9^{\mathrm{a}, \mathrm{b}}$ & $85.2 \pm 16.8^{b}$ & $15.3 \pm 4.2^{\mathrm{a}}$ & $18.9 \pm 16.7^{\mathrm{a}, \mathrm{b}}$ \\
\hline Non-AD degenerative $(n=7)$ & $65.4 \pm 9.1^{\mathrm{a}, \mathrm{b}}$ & $27.6 \pm 1.6$ & $3.9 \pm 4.1^{\mathrm{b}}$ & $85.4 \pm 19.1^{\mathrm{b}}$ & $14.0 \pm 3.2^{\mathrm{b}}$ & $27.6 \pm 30.1^{\mathrm{a}}$ \\
\hline Neurological $(n=25)$ & $61.5 \pm 10.9^{b}$ & $27.2 \pm 3.0$ & $4.1 \pm 2.8^{b}$ & $92.0 \pm 12.1^{\mathrm{a}, \mathrm{b}}$ & $12.8 \pm 2.8^{\mathrm{b}}$ & $12.0 \pm 14.3^{\mathrm{b}, \mathrm{c}}$ \\
\hline Medical $(\mathrm{n}=11)$ & $71.5 \pm 6.0^{\mathrm{a}}$ & $26.0 \pm 3.3$ & $3.8 \pm 1.9^{\mathrm{b}}$ & $97.7 \pm 3.9^{\mathrm{a}}$ & $13.8 \pm 2.7^{\mathrm{b}}$ & $3.70 \pm 5.6^{\mathrm{c}}$ \\
\hline Mixed $(\mathrm{n}=24)$ & $65.5 \pm 11.6^{\mathrm{a}, \mathrm{b}}$ & $26.9 \pm 2.2$ & $4.1 \pm 2.2^{\mathrm{b}}$ & $85.9 \pm 17.4^{\mathrm{b}}$ & $15.3 \pm 4.2^{\mathrm{a}}$ & $10.9 \pm 14.4^{\mathrm{b}, \mathrm{c}}$ \\
\hline CIND NOS $(n=66)$ & $62.5 \pm 10.6^{\mathrm{a}, \mathrm{b}}$ & $27.2 \pm 3.1$ & $4.5 \pm 3.9^{\mathrm{a}, \mathrm{b}}$ & $96.7 \pm 6.8^{\mathrm{a}}$ & $13.4 \pm 3.2^{\mathrm{b}}$ & $7.2 \pm 14.7^{b, c}$ \\
\hline $\mathrm{p}$ value & $<0.001$ & 0.95 & 0.004 & 0.001 & 0.004 & $<0.0001$ \\
\hline
\end{tabular}

The $\mathrm{p}$ values are obtained by ANCOVA with effect of age, sex, and education included in the model.

${ }^{\mathrm{a}-\mathrm{c}}$ Denote groups that are significantly different from each other by post-hoc Student-Newman-Keuls test with $\mathrm{p}<0.05$.

Table 3. Outcome of CIND at 2 years follow-up by CIND etiologic subtypes

\begin{tabular}{llccc}
\hline CIND subtypes & $\begin{array}{l}\text { Reverters } \\
(\mathrm{NCI})\end{array}$ & $\begin{array}{l}\text { Stable } \\
\text { CIND }\end{array}$ & $\begin{array}{l}\text { Progressors } \\
\text { (dementia) }\end{array}$ & Total \\
\hline Pre-AD & $2(4.4)$ & $25(55.6)$ & $18(40.0)$ & $45(30.8)$ \\
Vascular (VCI-ND) & $2(8.0)$ & $13(52.0)$ & $10(40.0)$ & $25(17.1)$ \\
Psychiatric & $4(19.0)$ & $12(57.1)$ & $5(23.8)$ & $21(14.4)$ \\
Non-AD degenerative & $1(33.3)$ & $1(33.3)$ & $1(33.3)$ & $3(2.1)$ \\
Other neurologic & $0(0.0)$ & $5(71.4)$ & $2(28.6)$ & $7(4.8)$ \\
Other medical & $0(0.0)$ & $2(40.0)$ & $3(60.0)$ & $5(3.4)$ \\
Mixed & $1(14.3)$ & $4(57.1)$ & $2(28.6)$ & $7(4.8)$ \\
CIND NOS & $10(30.3)$ & $15(45.5)$ & $8(24.2)$ & $33(22.6)$ \\
\hline Total & $20(13.7)$ & $77(52.7)$ & $49(33.6)$ & $146(100)$ \\
\hline
\end{tabular}

Figures in parentheses are percentage.

Percentages of reverters, stable CIND, and progressors represent proportions within each CIND subclass (within rows), while percentages in last column represent proportions of total (within column). loss. However, even when these two sites were excluded in our subsequent analyses, the findings were the same.

We evaluated baseline demographics and test scores of the CIND etiologic subtypes (table 2). The mean MMSE scores were similar across all groups. The VCI-ND and medical CIND were significantly older than the neurological and psychiatric CIND, and those with VCI-ND had the most co-morbid problems. The medical CIND and the CIND NOS group were the least impaired at baseline based on the DAD. The non-AD degenerative CIND had the highest NPI scores, higher even than those of the psychiatric CIND subjects.

Of the 146 subjects who completed a second annual assessment, 49 (34\%) progressed to dementia, while 20
(14\%) reverted to NCI, and 77 (52\%) remained stable. The outcome varied by etiologic subtype (table 3 ). The preAD group was the largest, and with VCI-ND, had the highest rate of conversion to dementia over 2 years at $40.0 \%$ each. By contrast, CIND NOS had the highest rate of reversion (30\%) to NCI. The numbers in the etiologic subtypes of non-AD degenerative, other neurologic, other medical, and mixed CIND are too small to make meaningful inferences. Overall, patients who progressed to dementia were significantly older (mean age $=71.1, \mathrm{SD}=$ 8.7) and had lower MMSE (mean MMSE = 26.2, SD = 1.72) at baseline compared to the stable CIND (mean age $=64.3, \mathrm{SD}=10.1 ;$ mean $\mathrm{MMSE}=28.1, \mathrm{SD}=1.7)$ and reverters to $\mathrm{NCI}$ (mean age $=59.1, \mathrm{SD}=9.1$; mean 
Table 4. Outcome of CIND progressors based on CIND subclassification

\begin{tabular}{|c|c|c|c|c|c|c|}
\hline \multirow[t]{2}{*}{ CIND subclasses } & \multicolumn{5}{|c|}{ Primary cause of dementia after conversion } & \multirow[t]{2}{*}{ Total } \\
\hline & $\mathrm{AD}$ & $\mathrm{VaD}$ & FTD & $\mathrm{PDD} / \mathrm{LBD}$ & Other & \\
\hline Pre-AD & $18(100)$ & - & - & - & - & $18(36.7)$ \\
\hline Vascular (VCI-ND) & $3(30)$ & $5(50)$ & - & $2(20)$ & - & $10(20.4)$ \\
\hline Psychiatric & $3(75)$ & - & $1(20)$ & - & $1(20)$ & $5(10.2)$ \\
\hline Non-AD degenerative & - & - & $1(100)$ & - & - & $1(2.0)$ \\
\hline Other neurologic & $1(50)$ & - & $1(50)$ & - & - & $2(4.1)$ \\
\hline Mixed CIND & $2(100)$ & - & - & - & - & $2(4.1)$ \\
\hline Other medical & $3(100)$ & - & - & - & - & $3(6.1)$ \\
\hline NOS & $5(62.5)$ & $1(12.5)$ & $2(25.0)$ & $0(0.00)$ & $0(0.00)$ & $8(16.3)$ \\
\hline Total (\% of Total) & $35(71.4)$ & $6(12.2)$ & $5(10.2)$ & $2(4.1)$ & $1(2.0)$ & 49 (100) \\
\hline
\end{tabular}

Figures in parentheses are percentage.

$\mathrm{AD}=$ Alzheimer disease; FTD = frontotemporal dementia LBD = Lewy body dementia PDD = Parkinson disease with dementia; $\mathrm{VaD}=$ vascular dementia.

MMSE $=28.8, \mathrm{SD}=1.2$ ). However, the sex distribution, level of education, and the baseline total scores on the FRS, DAD, and NPI did not differ significantly among the three outcomes (progressors, stable CIND, and reverters to $\mathrm{NCI}$ ).

Although the majority (71\%) of CIND progressors across all subtypes developed probable $\mathrm{AD}$ as the primary cause of their dementing illness, patients who progressed to dementia followed different patterns, depending on their etiologic subtype (table 4). Within the pre-AD CIND, all progressors developed probable AD by NINCDS-ADRDA criteria. For the VCI-ND, 50\% developed $\mathrm{VaD}$, while $30 \%$ were subsequently diagnosed with AD. Subjects who progressed to dementia had the highest rates of decline on the standard assessments, and those who did not progress showed the least (data not shown). The CIRS indicated significantly more medical decline in subjects with VCI-ND (mean change in CIRS $=-0.53, \mathrm{SD}=1.0$ ) and psychiatric CIND (mean change in CIRS $=-0.58, \mathrm{SD}=2.2$ ), while those with CIND NOS showed an improvement (mean change in CIRS $=+1.0, \mathrm{SD}=2.8$ ). The changes in DAD, FRS and NPI across the CIND subtypes were not significantly different.

ApoE genotype was available for 74 out of 146 CIND subjects. ApoE $\varepsilon 4$ carriers were much more likely to progress to dementia (21/31-67\%) compared to the stable CIND (10/35-29\%) and reverters (1/8-12.5\%). All homozygous $\varepsilon 4$ subjects $(7 / 7)$ converted to dementia at 2 -year follow-up. Among non- $\varepsilon 4$ carriers, $76 \%$ (32/42) remain stable or improved to NCI. The odds ratio of progression in $\varepsilon 4$ carriers compared to non- $\varepsilon 4$ carriers is 6.72 (95\% $\mathrm{CI}=2.93-11.9, \mathrm{p}=0.001$ ).

\section{Discussion}

We analyzed the outcome of CIND patients referred to specialized dementia clinics across Canada. The data allow for a comparison of CIND as identified and followed in referral dementia clinics with its prevalence and outcome in Canadian population-based epidemiological studies $[7,10,25,26]$. In the population-based studies of CSHA, CIND had a higher prevalence (16.8\%) [7] than the more specified MCI $(2.8-4 \%)[25,27]$, reflecting the more restrictive definition of MCI $[1,2,28]$ contrasting with the more inclusive nature of CIND. Data from this study show that CIND represents an important proportion $(30 \%)$ of all subjects referred to specialized memory disorder clinics [12].

The CIND group was heterogeneous in both cause and prognosis, the two were only incompletely related. Although many progressed to dementia (34\% over 2 years), most (52\%) remained stable at 2 years, with an appreciable $14 \%$ improving to be classified as NCI. Our data also show that our CIND subjects have one of the highest rates of conversion to dementia ( $34 \% / 2$ year) that has been reported to date, as compared to other population-based studies (ranging from 11 to $16 \% / 2-3$ year [25] to $19.3 \% / 5$ year [10]). The ACCORD CIND also have a lower rever- 
sion to normal rate (13.9\%/2 year) compared to some of the population-based estimates with return to normal rates of $40-55 \%$ over $2-3$ years $[10,25,27]$. These findings likely reflect on the referral process in the clinical setting. In Canada, patients are referred to specialized memory disorder clinics when their symptoms are sufficiently concerning to either themselves, their families, their family physicians, or their community specialists. This differs from the epidemiological studies where CIND is identified through testing of random population samples, where patient symptoms are not mandatory and where cognitive impairment may be incidentally picked up with cognitive tests. In the centers of the ACCORD, a screening test is often required as part of the referral process. Even though there is no specific 'cutoff' for referral, patients often exhibit some demonstrable objective impairment at the time a referral is made. It is also possible that CIND may be more advanced or more unstable when the referral is requested.

This broad-based approach of CIND allows for inclusion of all subjects who fall between the spectrum of normal cognition and dementia. This is also more applicable to the clinician in the usual care settings where amnestic MCI may account for only $25 \%$ of their CIND subjects. In this study, we have furthered the efforts to subclassify CIND into etiologic subtypes [7, 12]. The pre-AD CIND represented $30 \%$ of the total CIND population - a finding similar to the PAQUID cohort [27] and the CSHA [7], with MCI representing about $27-33 \%$ of all CIND subjects. However, it is not only the pre-AD subtype that converts to dementia. Rather our data demonstrates that despite the clinicians effort to classify the CIND patients into etiologic subtypes, all CIND subtypes can progress to dementia, with high conversion rates as well in the vascular and medical CIND, and lower but still sizable rates in the psychiatric CIND and CIND NOS.

Our data suggest that etiologic subclassification can have prognostic value in predicting the outcome of CIND. All of the pre-AD group who converted to dementia were classified as having clinically probable $\mathrm{AD}$. This indicates that the pre-AD CIND construct, although not sensitive, is a specific predictor of $\mathrm{AD}$. It also suggests that in the future, it may be possible to refine the clinical diagnostic criteria of early AD to allow inclusion of those with pre$\mathrm{AD}$ that have identifiable AD symptoms but not yet sufficiently advanced for formal diagnosis by criteria, perhaps with the aid of forthcoming biomarkers. The pathway to earlier diagnosis for this pre-AD group may reside within the patterns of early higher order functional impairments particularly in the domain of executive dys- function. Both the psychiatric and NOS groups stand out for their high rates of reversion to NCI and for their least predictable outcomes.

There are however, certain important limitations to our study. A sizable number of CIND subjects $(\mathrm{n}=190)$ were lost to follow-up at 2 years from the 342 subjects with CIND at entry. The loss to follow-up within our CIND group was proportional to the loss to follow-up rates in our NCI and dementia groups. Two sites were responsible for over $50 \%$ of the loss to follow-up, suggesting variability between sites regarding recontact efforts and techniques. However, when subjects from these two sites where excluded from other analyses, our findings remain the same. In considering the potential bias introduced by those lost to follow-up within our CIND group, we compared the baseline characteristics of missing subjects with those who completed their 2 year assessments, finding that that their mean MMSE at entry was significantly lower, and that they were on average more impaired on their global staging, their level of neuropsychiatric symptoms, and their functional assessments. On this basis, we speculate that if there is bias introduced with this lost to follow-up, it is likely to be conservative, underestimating the rate of progression to dementia of the CIND subjects. In comparing the lost to follow-up group with the other CIND groups by outcome, we have noted that their age and MMSE were most similar to the stable CIND, and intermediate between the progressors and reverters to NCI. This again would suggest that the lost to follow-up group likely consisted of a mix of reverters, stable CIND, and progressors. Unfortunately, the number of loss to follow-up also lead to relatively small numbers in some of the CIND subtypes (i.e. non-AD degenerative CIND, medical CIND, and other neurological CIND) limiting any inference from these categories. We are currently conducting a longer-term (6 year) follow-up with the intention of re-engaging the lost to follow-up subgroup to allow for a larger CIND subgroup analysis.

As in the CSHA cohort [29,30], we found that older age and a lower MMSE score at baseline are strong predictors of progression to dementia, probably because these subjects are at a more advanced stage of their condition. We also showed that homozygous ApoE $\varepsilon 4$ status, despite the clinically heterogeneous presentation of CIND, is a strong predictor of conversion to dementia, of which the majority were $\mathrm{AD}$ and $\mathrm{VaD}$. Interestingly, there was no obvious distinction between progressors and reverters of CIND by other clinical rating scales at baseline, perhaps reflecting the large variability within each group. However, when we examined the change in these clinical 
scales in the 2-year follow-up, there was significant decline in the progressors' functional abilities by DAD, FRS, and NPI, confirming the responsiveness and clinical utility of these scales in measuring progression of dementia.

The current study provides some insight into the natural history of CIND in the clinical setting. CIND is heterogeneous and dynamic. Subclassification of CIND by etiologic syndromes assists with the understanding of the various causes of CIND, helps with predicting the outcome of CIND, and may ultimately identify pathways to specifically manage each subtypes of CIND.

\section{Acknowledgements}

This research was funded by the Medical Research Council of Canada (now CIHR) and the Pharmaceutical Manufacturers Association of Canada, MRC PMAC program (grant-in-aid No. PA14197). Sponsorship from the PMAC was obtained from Sandoz, Smithkline Beecham, Pfizer Canada Inc., Hoffman LaRoche Ltd., Janssen Pharmaceutical Inc., and Astra. Dr. Hsiung was supported by post-doctoral fellowships from the Michael Smith Foundation for Medical Research (BC Canada) and the Arthur and June Willms fellowship through the Brain Research Center UBC, Canada. Dr. Rockwood receives career support from the CIHR, and from the Dalhousie Medical Research Foundation as Kathryn Allen Weldon Professor of Alzheimer Research. The authors would like to thank all of the subjects who participated in the study, and the study staffs at each of the participating centers. The authors would also like to acknowledge Ms. Sandra Tam for her assistance in preparation of the manuscript, and Dr. Claudia Jacova for her comments on the manuscript.

\section{Appendix 1.}

Diagnostic Checklist for NCI, CIND, and dementia (adapted from the DSM-III-R diagnostic criteria for dementia) [8]

\begin{tabular}{l} 
Criteria \\
1 There is impairment in \\
a short-term memory, or \\
b long-term memory \\
\hline 2 There is impairment in at least one of the following higher \\
cortical function such as: \\
a insight and judgement \\
b language problems or aphasia \\
c apraxia \\
d agnosia \\
e constructional difficulty \\
f personality change
\end{tabular}

3 Criteria 1 or 2 interfere with at least one of (a) work or IADL a social activities b relationships with others

4 Impairment occur outside episodes of delirium

5 No evidence (from history, physical examination) of specific organic factor(s) that can be etiologically related to the disturbance

6 No other mental disorders (such as major depreion) that could explain the change in cognitive function 'no'.

For 'No Cognitive Impairment' (NCI), criteria 1-3 must be all

For Dementia, all criteria 1-6 must be 'yes'.

For 'Cognitively Impaired Not Demented' (CIND) at least one of criteria 1 or 2 is 'yes', but not all criteria 1-6.

\section{References}

1 Petersen RC, Smith GE, Waring SC, et al: Mild cognitive impairment: clinical characterization and outcome. Arch Neurol 1999; 56:303-308.

-2 Petersen RC, Doody R, Kurz A, et al: Current concepts in mild cognitive impairment. Arch Neurol 2001;58:1985-1992.

3 Crook TH, Bartus RT, Ferris SH, et al: Ageassociated memory impairment: Proposed diagnostic criteria and measure of clinical change - Report of a National Institute of Mental Health Work Group. Dev Neuropsychol 1986;2:261-276.

-4 Blackford R, La Rue A: Criteria for diagnosing age-associated memory impairment: proposed improvements from the field. Dev Neuropsychol 1989;5:295-306.
5 Crook TH, Larrabee GJ, Youngjohn JR: Diagnosis and assessment of age-associated memory impairment. Clin Neuropharmacol 1990;13(suppl 3):S81-S91.

6 Levy R: Aging-associated cognitive decline. Working Party of the International Psychogeriatric Association in Collaboration with the World Health Organization. Int Psychogeriatr 1994;6:63-68.

7 Graham JE, Rockwood K, Beattie BL, et al: Prevalence and severity of cognitive impairment with and without dementia in an elderly population. Lancet 1997;49:1793-1796.

8 American Psychiatric Association: Diagnostic and Statistical Manual of Mental Disorders: DSM-III-R. Washington, DC, American Psychiatric Association, 1987.
9 CSHA: Canadian study of health and aging: study methods and prevalence of dementia. CMAJ 1994;150:899-913.

10 Tuokko H, Frerichs R, Graham J, et al: Fiveyear follow-up of cognitive impairment with no dementia. Arch Neurol 2003;60:577582

11 Solfrizzi V, Panza F, Colacicco AM, et al: Vascular risk factors, incidence of MCI, and rates of progression to dementia. Neurology 2004;63:1882-1891.

12 Feldman H, Levy AR, Hsiung GY, et al: A Canadian cohort study of cognitive impairment and related dementias (ACCORD): study methods and baseline results. Neuroepidemiology 2003;22:265-274.

13 Petersen RC: Mild cognitive impairment as a diagnostic entity. J Intern Med 2004;256: 183-194. 
-14 Rockwood K, Davis H, MacKnight C, et al: The Consortium to Investigate Vascular Impairment of Cognition: methods and first findings. Can J Neurol Sci 2003;30:237243.

-15 McKhann G, Drachman D, Folstein M, et al: Clinical diagnosis of Alzheimer's disease: report of the NINCDS-ADRDA Work Group under the auspices of Department of Health and Human Services Task Force on Alzheimer's Disease. Neurology 1984;34:939-944.

16 Lund and Manchester Groups: Clinical and neuropathological criteria for frontotemporal dementia. J Neurol Neurosurg Psychiatry 1994;57:416-418.

- 17 McKeith IG, Galasko D, Kosaka K, et al., Consensus guidelines for the clinical and pathologic diagnosis of dementia with Lewy bodies (DLB): report of the consortium on DLB international workshop. Neurology 1996;47:1113-1124

18 Folstein MF, Robins LN, Helzer JE: The Mini-Mental State Examination. Arch Gen Psychiatry 1983;40:812.
19 Crockett D, Tuokko H, Koch W, et al: The assessment of everyday functioning using the Present Runctioning Questionnaire and the Functional Rating Scale in elderly samples. Clin Gerontol 1989;8:3-25.

20 Tuokko H, Crockett D, Beattie BL, et al: The use of rating scales to assess psychosocial functioning in demented patients. J Clin Exp Neuropsychol 1985;7:615.

21 Parmelee PA, Thuras PD, Katz IR, et al: Validation of the Cumulative Illness Rating Scale in a geriatric residential population. J Am Geriatr Soc 1995;43:130-137.

22 Linn BS, Linn MW, Gurel L: Cumulative illness rating scale. J Am Geriatr Soc 1968;16: 622-626.

23 Gelinas I, Gauthier L, McIntyre M, et al: Development of a functional measure for persons with Alzheimer's disease: the disability assessment for dementia. Am J Occup Ther 1999;53:471-481.

24 Cummings JL, Mega M, Gray K, et al: The Neuropsychiatric Inventory: comprehensive assessment of psychopathology in dementia. Neurology 1994;44:2308-2314.
25 Ganguli M, Dodge HH, Shen C, et al: Mild cognitive impairment, amnestic type: an epidemiologic study. Neurology 2004;63:115121.

26 Unverzagt FW, Gao S, Baiyewu O, et al: Prevalence of cognitive impairment: data from the Indianapolis Study of Health and Aging. Neurology 2001;57:1655-1662.

-27 Larrieu S, Letenneur L, Orgogozo JM, et al: Incidence and outcome of mild cognitive impairment in a population-based prospective cohort. Neurology 2002;59:1594-1599.

-28 Fisk JD, Merry HR, Rockwood K: Variations in case definition affect prevalence but not outcomes of mild cognitive impairment. Neurology 2003;61:1179-1184.

29 Hogan DB, Ebly EM: Predicting who will develop dementia in a cohort of Canadian seniors. Can J Neurol Sci 2000;27:18-24.

30 Hsiung GY, Sadovnick AD, Feldman H: Apolipoprotein E e4 genotype as a risk factor for cognitive decline and dementia: data from the Canadian Study of Health and Aging. CMAJ 2004;171:863-867. 